\title{
LA USMC Y SU INFLUENCIA \\ SOBRE LA INDUSTRIA DEL CALZADO \\ DE ESTADOS UNIDOS, LATINOAMÉRICA Y EUROPA \\ EN LA PRIMERA MITAD DEL SIGLO XX. ¿UN MONOPOLIO EFICIENTE?*
}

\section{THE USMC AND ITS INFLUENCE ON THE FOOTWEAR INDUSTRY OF THE UNITED STATES, LATIN AMERICA AND EUROPE IN THE FIRST HALF OF THE TWENTIETH CENTURY. AN EFFICIENT MONOPOLY?}

\author{
José A. Miranda \\ Universidad de Alicante, España, miranda@ua.es
}

Resumen. Este artículo analiza la estrategia de la United Shoe Machinery Company, empresa que dominó el mercado mundial de maquinaria para calzado en la primera mitad del siglo Xx, su penetración en Latinoamérica y en el resto del mundo y los procesos judiciales antimonopolio que sufrió en Estados Unidos. A pesar de su posición de práctico monopolio, la empresa tuvo un desempeño eficiente que impulsó la innovación tecnológica y la modernización de la industria del calzado en muchos países.

Palabras clave: monopolio; industria del calzado; mercados disputados; leyes antimonopolio; estrategia empresarial.

Abstract. The United Shoe Machinery Company dominated world market for footwear machinery in the first half of the twentieth century. This article analyzes the company's strategy, its penetration in Latin America and the rest of the world, and the impact on the company of antitrust lawsuits in the United States. Despite its practical monopoly, the company had an efficient performance, which fueled technological innovation and modernization of footwear industry in many countries.

Key words: monopoly; footwear industry; contestable markets; antitrust laws; business strategy.

Fecha de recepción: 20 de marzo de 2014. Fecha de aceptación: 30 de junio de 2014.

* Este trabajo se ha realizado con el apoyo del Ministerio de Economía y Competitividad del Gobierno de España, a través del proyecto HAR2012-33298.

Am. Lat. Hist. Econ., año 22, núm. 2, mayo-agosto, 2015, pp. 71-100 


\section{INTRODUCCIÓN}

$\mathrm{L}$ a teoría económica neoclásica afirma que los monopolios, es decir, las situaciones opuestas a los mercados perfectamente competitivos, se caracterizan porque el monopolista puede determinar el precio al decidir qué cantidad produce. En estas circunstancias, para maximizar sus beneficios, la empresa con poder de monopolio reducirá la cantidad final producida y elevará el precio en comparación con lo que sucedería en una situación de mercado competitivo. Los precios del monopolio se diferenciarán más de los de competencia cuanto menor sea la elasticidad de la demanda respecto del precio del bien producido. Por tanto, el monopolio no permite al mercado funcionar eficientemente y tiene un costo social. Este razonamiento justifica que los poderes públicos intervengan activamente para combatir las situaciones de monopolio y fomentar la competencia.

Estados Unidos, a finales del siglo XIX, fue uno de los primeros países en adoptar medidas antimonopólicas al elaborar una legislación específica para ello. El gran tamaño del mercado estadunidense acentuó la tendencia hacia la gran empresa generada por la segunda revolución tecnológica y el poder político intentó limitar el fenómeno al aprobar la Ley Sherman en 1890 y la Ley Clayton en 1914. La aplicación de ambas leyes dio lugar a complejos procesos judiciales, en los que se vieron envueltas algunas de las principales empresas del país. La United Shoe Machinery Company (en adelante USMC) fue una de estas empresas donde los procesos que se abrieron contra ella, especialmente el iniciado en 1947, se convirtieron en una referencia ineludible dentro de la jurisprudencia sobre la materia. Se trata de un caso interesante, donde se aprecia que la realidad no siempre se ajusta a las simplificaciones de la teoría económica.

Si se define el mercado de maquinaria para calzado como el de las máquinas específicamente realizadas para el proceso de fabricación de este tipo de artículo, cuya oferta está integrada por las empresas especializadas en la producción de estas máquinas y su demanda por las empresas fabricantes de calzado, y si se mide la participación de las empresas oferentes a través de la proporción de unidades físicas de maquinaria en activo suministradas por cada una de estas empresas, se puede afirmar que la USMC logró hacerse con una posición ampliamente dominante en este mercado, cercana al monopolio, en Estados Unidos y en muchos otros países en la primera mitad del siglo XX (Kaysen, 1956).

Esta situación impidió el desarrollo de otras empresas de maquinaria con esta especialización y canalizó un notable flujo de recursos desde las fábricas de calzado hacia la compañía. Sin embargo, este artículo intenta mostrar que, frente a lo que pronostica la teoría económica, ese dominio 
fue acompañado de un desempeño eficiente, que facilitó la modernización tecnológica de la industria del calzado en muchos países.

En una primera parte, se sintetiza el origen y la trayectoria de la USMC, además de mostrar los rasgos principales de su estrategia empresarial, entre los que sobresale el sistema de arrendamiento de la maquinaria. También se resume la situación en la que se encontraba la industria del calzado en Europa y Latinoamérica a principios del siglo Xx y la influencia que en ella tuvo la USMC. A continuación, se expone cómo afectó a la compañía la política antimonopolio de Estados Unidos. En el apartado siguiente se recoge la revisión que diversos autores han realizado, desde el punto de vista económico, de las sentencias contra la USMC. Esto da pie a un análisis de las consecuencias que tuvo para la industria la posición monopolística de la compañía y las medidas dictadas por los tribunales para contrarrestarla. Finalmente, se concretan las conclusiones del trabajo.

\section{LA USMC Y LA MODERNIZACIÓN DE LA INDUSTRIA DEL CALZADO}

La industria del calzado no comenzó a mecanizarse verdaderamente hasta mediados del siglo XIX. Este retraso se debió principalmente a la dificultad técnica que suponía mecanizar el trabajo con la piel, material no homogéneo, pero también a la compleja forma del zapato, que requiere realizar movimientos curvos, y la necesidad de fabricar un producto diversificado, para ajustarse a las distintas tallas, y sujeto a los cambios de temporada y las modas. Fue en Estados Unidos donde se gestaron las principales innovaciones técnicas en esta industria y donde más rápidamente se difundieron.

Gracias a las aportaciones de un nutrido grupo de inventores, al acabar el siglo XIX ya se contaba con máquinas específicas para cada una de las múltiples tareas del proceso de fabricación del calzado, y la rápida aceptación de la nueva tecnología por parte de las empresas zapateras hizo que se creara una importante industria de maquinaria para el sector en Estados Unidos. El desarrollo de esta industria, a su vez, impulsó la generación de innovaciones y su difusión, de modo que a finales del siglo XIX la mayoría de las empresas zapateras estadunidenses tenía ya muy mecanizado casi todo su proceso de fabricación y la mejora de la productividad, respecto a décadas precedentes, había rebajado sustancialmente los costes del trabajo por unidad de producto (Hoover, 1937, p. 206).

Inicialmente surgieron numerosas empresas especializadas en la producción de maquinaria para una fase determinada de la fabricación de calzado. Sin embargo, estas empresas fueron diversificando progresivamente su oferta e intentaron desarrollar un sistema completo de maquinaria para todo el proceso. La estrategia comercial adoptada resultó clave 
para la rápida difusión de las innovaciones, ya que las empresas tendieron a crear extensas redes comerciales y de asistencia técnica, con delegaciones en las principales ciudades productoras de calzado, que aseguraban a los clientes el mantenimiento de las máquinas y facilitaban la formación de los operarios que iban a utilizarlas. Además, la maquinaria no era vendida, sino que se arrendaba, de modo que los fabricantes de calzado no tenían que realizar ninguna inversión importante en capital fijo para adoptar la nueva tecnología.

La aspiración de ofrecer un sistema integrado de maquinaria condujo a una mayor concentración empresarial. El principal exponente de este fenómeno fue la constitución de la United Shoe Machinery Company of New Jersey (USMC) en febrero de 1899. La compañía fue el resultado de la fusión de cinco empresas, tres de las cuales tenían una posición dominante en el mercado de sus respectivas especialidades: Goodyear Shoe Machinery Corporation, especializada en maquinaria para el calzado empalmillado; McKay Shoe Machinery Company, líder en maquinaria para la construcción de tacones y cierres metálicos, y Consolidated and McKay Lasting Machine Company, especializada en máquinas de montar. La cuarta empresa, Davey Pegging Machine Company, estaba especializada en una tecnología, la del "estaquillado", que ya comenzaba a quedar obsoleta. Finalmente, la quinta integrante de la fusión fue Eppler Welt Machine Company, una pequeña empresa que estaba introduciéndose en el sector. Los servicios técnicos de las compañías fusionadas se integraron en una sola red y, a partir de 1906, la firma concentró la producción en una nueva planta en Beverly, Massachusetts, con 3000 trabajadores, que era la mayor y más avanzada fábrica de maquinaria en Estados Unidos (Thomson, 1989).

La USMC logró ofrecer maquinaria para todas las operaciones básicas del proceso de producción del calzado, salvo para coser las partes del empeine. Al constituirse, su participación en el mercado de la maquinaria superaba el 70\% en Estados Unidos. Además de esto, una estrategia de amplia reinversión de los beneficios le permitió crecer con rapidez: en diez años, el valor de los activos de la compañía pasó de 15000000 de dólares a más de 40000000 de dólares. Además, la USMC amplió su oferta de maquinaria al tiempo que reforzó su control del mercado mediante una inversión fuerte en investigación, a través de la adquisición de patentes, de otras empresas menores y contratación de personal de I+D (investigación y desarrollo) procedente de compañías competidoras. Fue una de las primeras compañías estadunidenses en establecer un departamento de investigación e invirtió anualmente en él alrededor de medio millón de dólares en los quince años siguientes a su constitución. En 1920 su departamento de investigación multiplicaba por cinco en personal y por ocho en gasto el tamaño medio de estos departamentos en las empresas estadunidenses. 
También adquirió más de medio centenar de patentes y activos de otras empresas en su primera década de existencia. Estas adquisiciones de empresas y la creación de filiales produjeron una reorganización corporativa, con la constitución de la United Shoe Machinery Corporation en mayo de 1905 y bajo las leyes del estado de Nueva Jersey, que adquirió todas las acciones de la United Shoe Machinery Company. Doce años después, en diciembre de 1917, hubo una nueva reorganización y la United Shoe Machinery Company de Nueva Jersey se fusionó con la Corporation (Kaysen, 1956, pp. 6-11).

La elevada inversión en I+D y la adquisición de activos de la competencia, incluso fuera de Estados Unidos, continuó en las décadas siguientes. Hacia 1930, la empresa contaba con una plantilla de 271 empleados en su departamento de investigación e invertía anualmente en el mismo casi 2000000 de dólares. Veinte años después, tanto el número de investigadores como la inversión en este campo se habían más que duplicado y en la industria mecánica sólo la empresa automovilística General Motors poseía un departamento de investigación mayor. Esto permitió a la compañía aportar cerca de 40\% de todas las patentes de maquinaria para calzado registradas en Estados Unidos y ser una de las cinco compañías del país con mayor número de patentes. También desarrolló cuatro de las seis máquinas fundamentales incorporadas al sector entre 1925 y 1950 , según algunos expertos. Además, el departamento de investigación realizaba continuas mejoras en la maquinaria que en su mayoría no se patentaban. A lo largo de la vida activa de un modelo de máquina se introducían como promedio más de 190 modificaciones, de las que se patentaban menos de 5\% (Kaysen, 1956).

La cuota de mercado de la compañía siguió incrementándose de modo que a finales de la década de 1940 la USMC ya concentraba más de 90\% del mercado de buena parte de la maquinaria fundamental y casi $75 \%$ de la maquinaria auxiliar en la fabricación de calzado en Estados Unidos (Kaysen, 1956). El valor de los activos de la compañía hacia 1950 superaba los 100000000 de dólares. Su actividad no se limitó a la fabricación y comercialización de la maquinaria para calzado, sino que la USMC también se dedicó a la maquinaria para la industria del curtido y a la producción y distribución de complementos para las máquinas, de accesorios para la fabricación de calzado y de componentes y materiales para calzado.

Desde un principio, la compañía continuó con la práctica de arrendar la mayoría de la maquinaria e incluir el servicio técnico en el arrendamiento, sin cargo separado (instalación de las máquinas, instrucción de los operarios y reparación de la maquinaria estropeada). A mediados de la década de 1940, 80\% de la maquinaria de la USMC que era utilizada en las empresas estaba en arrendamiento y sólo $20 \%$ de ellas había sido vendido. 
Solían venderse las máquinas sencillas, de carácter auxiliar y algunos modelos antiguos. Estas ventas sólo proporcionaban aproximadamente 2\% de los ingresos de la compañía. Inicialmente los contratos de arrendamiento se realizaban por un plazo de 20 años, pero a partir del segundo proceso por prácticas monopolísticas contra la USMC el plazo se acortó a diez años y tras la sentencia de 1953 se redujo a cinco. El pago solía estar en función del uso de la maquinaria o bien era una cantidad mensual fija, aunque también había máquinas en las que se combinaban ambos tipos de tarifas. Los contratos incluían una variedad de cláusulas que limitaban la libertad de utilizar maquinaria que no fuese de la USMC e incluso obligaban a los arrendatarios a utilizar repuestos, complementos y materiales de la compañía, y que permitían a esta retirar la maquinaria si el arrendatario no alcanzaba un volumen de producción suficiente (British Boot Machinery Manufacturers' Association, c. 1919).

Ya antes de la constitución de la USMC, las empresas de maquinaria para calzado de Estados Unidos habían buscado la comercialización internacional de sus productos, estableciendo puntos de venta y empresas de distribución en otros países, principalmente en Europa. La USMC dio un impulso sustancial a esta expansión exterior y, por lo tanto, a la difusión internacional de la tecnología estadunidense para el calzado, principalmente en Europa, pero también en Latinoamérica y en los dominios británicos en América, Oceanía y África. El impulso dado por la USMC a la penetración de la maquinaria estadunidense para el calzado en los mercados internacionales puede verse reflejado en la evolución de las exportaciones: entre 1899 y 1906, es decir, en los siete primeros años de vida de la USMC, el valor de las exportaciones de este tipo de maquinaria desde Estados Unidos creció a una tasa acumulativa media superior a 8\% anual, y en 1912, cuando estas exportaciones alcanzaron el máximo del periodo anterior a la primera guerra mundial, su valor multiplicaba por más de dos el registrado en 1898, previo a la constitución de la compañía (Church, 1968).

La compañía fue capaz de trasladar al exterior, mediante una extensa red de delegaciones y empresas filiales, la estrategia de comercialización que había permitido la rápida difusión de la maquinaria en su país de origen y consiguió así facilitar enormemente la mecanización de la industria del calzado en otros territorios. Las primeras empresas filiales de la USMC se establecieron, el mismo año de constitución de la compañía, en Canadá y en el Reino Unido. En 1900 se crearon empresas similares en Francia y Alemania, y cuando se inició la primera guerra mundial ya se habían instalado empresas de este tipo en Italia, Austria, Argentina y México. Por entonces, la USMC contaba también con delegaciones o depósitos de maquinaria en Suiza, Dinamarca, Noruega, Suecia, Bélgica, Australia, Nueva Zelanda, Chile, Brasil y España. De esta forma, la USMC consiguió 
trasladar a sus principales mercados exteriores la posición dominante que disfrutaba en el mercado estadunidense. En Europa, por ejemplo, en vísperas de la primera guerra mundial, ya controlaba $80 \%$ de la maquinaria instalada en la industria del calzado británica, alrededor de las tres cuartas partes de la instalada en Francia y 90\% de la existente en Italia y en España (Butman, 1912a, 1912b, 1913a, 1913b).

La expansión internacional de la USMC fue de la mano del desarrollo de la industria del calzado en los distintos países y contribuyó decisivamente a la modernización técnica del sector e incremento de la productividad. Siguiendo el ejemplo estadunidense, Reino Unido fue el primer país europeo en disponer de una industria del calzado moderna y competitiva internacionalmente. A pesar de que Inglaterra contaba con una industria de maquinaria para calzado importante y mantenía un estrecho contacto con las empresas estadunidenses de maquinaria desde décadas atrás, la intervención de la USMC también fue decisiva. Su filial en el país, la British United Shoe Machinery Company, intensificó la influencia estadunidense sobre la industria del calzado británica y logró que la mayor parte de esta estuviese tan bien equipada tecnológicamente como las empresas de Estados Unidos. Con ello, la industria británica mejoró su competitividad y pudo frenar en los primeros años del siglo xx el acelerado crecimiento de las importaciones de calzado americano que se había producido en el Reino Unido desde las últimas décadas del siglo XIX (Church, 1968, pp. 247-254).

Tras la industria británica, las siguientes en modernizar sus estructuras y convertirse en destacadas exportadoras a principios del siglo Xx fueron las industrias de calzado alemanas y suizas. En Alemania el rápido avance de la mecanización no sólo se vio impulsado por la Deutsche Vereinigte Schuhmaschinen Gesellschaft, filial de la USMC establecida en Fráncfort, sino también por las dinámicas empresas alemanas de maquinaria para el calzado, entre las que destacaba Maschinenfabrik Moenus A. G. La industria del calzado alemán, bien equipada técnicamente y con salarios más bajos que los británicos y franceses, logró penetrar en los mercados exteriores desde principios del siglo XX y se convirtió en una gran exportadora a escala mundial desde mediados de la década de 1920 (Butman, 1912c; The Leather Trade's Review, 14 de noviembre de 1928, p. 908).

La industria suiza se benefició de la maquinaria de la USMC por medio de la delegación de esta empresa en Zúrich, pero recibió también maquinaria alemana y francesa. Casi la mitad de la producción del sector procedía de una sola compañía, Bally, que antes de la Gran Guerra fabricaba aproximadamente 3000000 de pares anuales, la mayor parte era calzado para mujer de calidades media y alta, destinado a los mercados europeos y de Latinoamérica (Butman, 1912a, pp. 27-39). 
En Francia, la United Shoe Machinery Company de France favoreció la introducción de maquinaria en la industria del calzado desde su creación en 1900 y la producción anual del sector se acercó a los 25000000 de pares ya antes de la Gran Guerra. Sin embargo, tanto la mecanización como la adopción del sistema fabril avanzaron en este país más lentamente que en Alemania o Suiza, y el sector continuó caracterizado por las pequeñas empresas de producción diversificada y una abundante utilización del trabajo a domicilio al menos hasta los años de la primera guerra mundial. El progreso fue todavía más lento en Italia, España y los territorios del imperio austrohúngaro. En Italia, la incorporación de maquinaria en las empresas de calzado se incrementó a partir de 1906, cuando la USMC mostró las posibilidades de esta tecnología en la Exposición Internacional celebrada en Milán, pero lo que verdaderamente acentuó el crecimiento y la modernización fue la introducción del sistema de arrendamiento de la maquinaria por la compañía estadunidense en 1912. No obstante, todavía en los años treinta había multitud de pequeños talleres, que realizaban más de un tercio de la producción total (Butman, 1913b, pp. 5-31; Fontana, 1998; Sabbatucci, 1998, 2001, 2007, 2010; Segreto, 1989).

También en España la industria del calzado se modernizó tardíamente. Según los informes del Departamento de Comercio de Estados Unidos, la fabricación mecanizada de calzado de cuero no alcanzaba los 5000000 de pares anuales antes de la primera guerra mundial (Butman, 1913a), mientras que la producción realizada básicamente a mano debía sumar al menos 3000000 más. La maquinaria de la USMC comenzó a llegar al país a través de la filial de la compañía en Francia, que pronto estableció una delegación en España, pero en 1917 se constituyó legalmente una filial española, encargada también del mercado portugués. Esta maquinaria fue esencial para incrementar la productividad del sector, pero la producción y el consumo de calzado de cuero en España se mantuvieron a un nivel bajo en comparación con la Europa avanzada. Antes del estallido de la guerra civil, la producción por habitante quizá era algo superior a lo que reflejan los cálculos estadunidenses (véase cuadro 1), pero sin duda estaba muy por debajo de la francesa (Bibiloni y Pons, 2001; Escartín, 2001; Manera, 2002; Miranda, 1998; Nadal, 1994; Ponce y Martínez, 2003; Valero, Navarro, Martínez y Amat, 1992).

En el imperio austrohúngaro la mecanización de la industria también avanzó muy lentamente antes de la Gran Guerra, de modo que la mayoría de la producción continuó realizándose manualmente. En el progreso hacia estructuras fabriles más avanzadas también resultó decisiva la influencia de la USMC, que instaló una empresa filial en Viena. A pesar de la baja productividad, la industria austriaca tenía una fuerte capacidad exportadora, especialmente de calzado para mujer, ligero y elegante 
(Butman, 1913b, pp. 32-54). Tras la Gran Guerra y la desintegración del imperio, fue el reciente Estado checoslovaco quien configuró una industria dinámica, gracias a la empresa Bata, que inundó los mercados internacionales de calzado de bajo precio (Miranda, 2009, pp. 3-6). Asimismo, en el primer tercio del siglo XX se configuraron industrias del calzado de cierta importancia, a pesar del reducido tamaño de sus mercados nacionales, en Bélgica y Holanda y, en un entorno económico totalmente distinto, en la URSS (véase cuadro 1).

La USMC también conquistó los mercados latinoamericanos. Antes de la segunda guerra mundial, sólo Brasil, Argentina, Chile, Cuba y México contaban con industrias del calzado de cuero de cierta importancia (véase cuadro 2). El resto de los países tenía una producción limitada, realizada artesanalmente debido al pequeño tamaño de su población, al bajo poder adquisitivo de la mayor parte de ella y al generalizado consumo de otros tipos de calzado de menor precio, como las alpargatas. En Argentina y Chile, la industria del calzado logró un tamaño destacado antes de la primera guerra mundial. Según el censo industrial, la ciudad de Buenos Aires, que concentraba el grueso de la producción argentina de calzado, contaba en 1908 con 223 fábricas de calzado de cuero con cerca de 7000 trabajadores, más de la mitad de ellos a domicilio, y con casi medio millar de talleres artesanos que realizaban zapatos a medida y para los que trabajaban más de 2000 personas. Ello convertía al calzado en la segunda manufactura de la ciudad por su volumen de empleo (Barbero, 2011; Butman, 1910a; Kabat, 2001).

En Chile, la industria del calzado creció con rapidez en el segundo lustro del siglo XX, también muy concentrada en la capital del país, donde se localizaba más de la mitad de las 50 fábricas de calzado en funcionamiento hacia 1910. Allí, como en Argentina, la mayoría de las principales empresas eran propiedad de inmigrantes europeos (Butman, 1910a). Brasil, por su parte, contaba en 1909 con 119 fábricas de calzado, aunque menos de la mitad estaban mecanizadas, y un gran número de talleres artesanales. En total, el sector empleaba en el país a más de 7000 trabajadores y se concentraba especialmente en Río de Janeiro y en los estados de São Paulo y Rio Grande do Sul (Butman, 1910b).

En Cuba, en cambio, la industria del calzado no comenzó a desarrollarse sino hasta los años de la primera guerra mundial. Todavía en 1910 sólo había tres fábricas mecanizadas y la mayoría del calzado consumido en la isla era importado de Estados Unidos y España (Butman, 1909). Una situación similar se daba en México, donde, a pesar de las elevadas tarifas arancelarias, las importaciones de calzado rondaban el millón de pares al año, casi $90 \%$ procedentes de Estados Unidos, y sólo existían trece fábricas propiamente dichas, aunque sí eran muy numerosos los pequeños talleres manuales (Butman, 1909). En conjunto, la industria latinoameri- 


\section{CUADRO 1. PRODUCCIÓN DE CALZADO DE CUERO EN PAÍSES EUROPEOS, 1936}

\begin{tabular}{lrr}
\hline País & $\begin{array}{c}\text { Producción } \\
\text { (miles de pares) }\end{array}$ & Pares/100 hab. \\
Reino Unido & 130000 & 276.12 \\
URSS & 90000 & 49.59 \\
Alemania & 76000 & 112.85 \\
Francia & 50000 & 119.30 \\
Checoslovaquia & 30000 & 208.52 \\
Italia & 30000 & 70.18 \\
Bélgica & 16500 & 198.44 \\
Holanda & 16000 & 187.88 \\
Polonia & 15000 & 49.23 \\
España & 14000 & 56.96 \\
Suecia & 10000 & 159.77 \\
Suiza & 8200 & 196.74 \\
Austria & 6000 & 88.78 \\
Grecia & 5250 & 76.24 \\
Dinamarca & 5148 & 138.31 \\
Hungría & 5000 & 55.27 \\
Irlanda & 4375 & 147.46 \\
Yugoslavia & 4125 & 26.36 \\
Bulgaria & 3150 & 48.69 \\
Noruega & 3000 & 103.31 \\
Finlandia & 2800 & 77.76 \\
Portugal & 2000 & 27.38 \\
Rumania & 1318 & 8.64 \\
\hline & & \\
\hline
\end{tabular}

${ }^{\text {a }}$ Los datos son a partir de 1935.

Fuente: elaboración propia con base en Schnitzer (1937).

cana del calzado estaba mucho menos desarrollada que la europea, pero en Argentina y Chile la estructura productiva era al menos tan moderna como la que se encontraba en Italia o España, de donde procedía una gran parte de los empresarios del sector instalados en estos dos países del sur de América.

La Gran Guerra y las políticas proteccionistas que se aplicaron tras ella y, especialmente, desde finales de la década de 1920, impulsaron la sustitución de importaciones y favorecieron el crecimiento del sector en 
Am. Lat. Hist. Econ., año 22, núm. 2, mayo-agosto, 2015, pp. 71-100

\section{CUADRO 2. PRODUCCIÓN DE CALZADO DE CUERO EN LOS PAÍSES LATINOAMERICANOS, 1936}

\begin{tabular}{lcc}
\hline País & $\begin{array}{c}\text { Producción } \\
\text { (miles de pares) }\end{array}$ & Pares/100 hab. \\
Brasil & 19000 & 50.1 \\
Argentina & 16000 & 120.7 \\
México & 5200 & 27.3 \\
Cuba & 4000 & 93.3 \\
Chile & 3600 & 76.5 \\
Venezuela & $2000^{\mathrm{a}}$ & 57.0 \\
Perú & 1200 & 19.9 \\
Uruguay & 1150 & 60.9 \\
Colombia & 1000 & 11.8 \\
Nicaragua & 500 & 66.7 \\
Ecuador & 460 & 20.5 \\
República Dominicana & 415 & 27.3 \\
Costa Rica & 350 & 62.5 \\
Bolivia & 325 & 12.7 \\
El Salvador & 270 & 17.4 \\
Guatemala & 265 & 13.1 \\
Haití & 265 & 10.1 \\
Paraguay & 225 & 22.2 \\
Panamá & 200 & 32.9 \\
Honduras & 100 & 9.4 \\
\hline
\end{tabular}

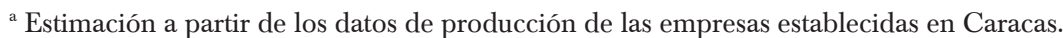
Fuente: elaboración propia con base en Schnitzer (1937).

Latinoamérica. A mediados de la década de 1930, la industria del calzado argentino había incrementado el número de trabajadores hasta 25000 y producía anualmente alrededor de 16000000 de pares de botas y zapatos de cuero, a los que sumaba 9000000 de pares de zapatillas y 29000000 de pares de alpargatas; por el contrario, las importaciones de calzado de cuero en el país, que antes de la Gran Guerra superaban el millón de pares, se habían reducido a poco más de 3000 pares (Brock, 1919, p. 25; Schnitzer, 1937, pp. 38-40). En Chile, el empleo llegó hasta 23000 trabajadores en 1930, cuando la capacidad productiva de las fábricas de calzado alcanzó los 4500000 pares y la de los pequeños talleres, 2500 000; sin embargo, la depresión económica redujo la producción a 3600000 pares 
en 1936. Las importaciones, que antes de la guerra habían rondado los 150000 pares, cayeron a menos de 500 pares en 1935 (Brock, 1918, p. 16; Schnitzer, 1937, pp. 46-47).

En Brasil, las exportaciones de calzado de cuero superaron por primera vez a las importaciones en 1934, cuando estas últimas se redujeron hasta los 1000 kilogramos de peso. La producción se acercó por entonces a los 19000000 de pares anuales (29000 000 si se incluyen las zapatillas y otros tipos de calzado), obtenidos en poco más de 250 fábricas mecanizadas y cerca de 8000 establecimientos de pequeño tamaño y producción fundamentalmente manual, que empleaban en conjunto a unos 13000 trabajadores. El crecimiento del sector se había concentrado especialmente entonces en el estado de São Paulo, donde se realizaba más de $40 \%$ de la producción del país (Schnitzer, 1937, pp. 43-46). En Cuba, las importaciones aún aportaban casi $60 \%$ del consumo de calzado hasta 1927, pero ese año se incrementaron notablemente las tarifas arancelarias sobre este producto, lo que dio lugar a un rápido descenso de las importaciones y a un crecimiento de la producción. En 1934 se fabricaron más de 4000000 de pares, casi el doble que siete años antes, y las compras en el exterior se redujeron en más de 98\%. Sin embargo, el sector todavía dependía mayoritariamente de los pequeños talleres manuales y sólo existían 18 fábricas mecanizadas (Schnitzer, 1937, pp. 18-19). También en México los pequeños talleres manuales constituían la mayoría de los más de 3000 establecimientos registrados para la fabricación de calzado y, mientras la producción aumentó hasta superar los 5000000 de pares anuales en 1935, las importaciones se redujeron a tan sólo 13000 pares (Schnitzer, 1937, pp. 24-27).

En términos absolutos, ningún país latinoamericano presentaba a mediados de la década de 1930 cifras de producción de calzado destacadas a escala mundial y sólo Brasil y Argentina tenían un volumen de producción comparable al de los países europeos de tamaño medio. Sin embargo, el conjunto de Latinoamérica, con una producción anual de más de 50000000 de pares, sí representaba un mercado significativo para las compañías suministradoras de maquinaria para la industria del calzado. Por otro lado, si se considera la producción en relación con la población, la industria del calzado argentina se situaba por encima no sólo de la industria de países del sur de Europa, como España o Italia, sino incluso de otros muy avanzados económicamente, como Francia o Alemania. Y también Cuba y Chile tenían cifras de producción por habitante superiores a las de la Europa meridional.

A inicios del siglo Xx, la maquinaria utilizada en las empresas de calzado latinoamericanas procedía en gran parte de Europa, especialmente de Francia (con la empresa Johnson et Fils como líder) y Alemania (particularmente de la empresa Moenus), pero también de Reino Unido y, en 
menor medida, de España. La USMC creó en 1902 una filial llamada United Shoe Machinery Company of South America para introducirse en la región. Primero se estableció en Buenos Aires y poco después en Santiago de Chile y Río de Janeiro. Su estrategia para desplazar a las importaciones de maquinaria europea consistió en trasladar a Latinoamérica su sistema de arrendamiento de maquinaria, con esta acción reducía considerablemente la inversión inicial que debían realizar las fábricas de calzado para mecanizarse, y en trasladar también su red de asistencia técnica, que aseguraba el mantenimiento de la maquinaria y el suministro de recambios y accesorios de manera mucho más eficiente y ágil.

Para demostrar el elevado rendimiento de su tecnología, la USMC instaló una línea de maquinaria completa para elaborar calzado empalmillado sin coste durante dos años en la empresa Bordás y Conte, de Buenos Aires, esto a cambio de utilizarla como modelo ante los otros fabricantes (Brock, 1919, pp. 50-51). A partir de entonces, la maquinaria estadunidense se impuso progresivamente, por lo que antes del inicio de la primera guerra mundial ya era mayoritaria en los principales países productores de calzado de la región y este predominio se acrecentó durante los años de la guerra, por la extraordinaria dificultad para importar maquinaria y repuestos desde Europa.

En Chile, por ejemplo, donde la United Shoe Machinery Company of South America abrió una delegación en 1908, Estados Unidos ya proporcionaba más de 70\% de las importaciones de maquinaria para calzado antes de la Gran Guerra, y hacia 1930, 90\% de la maquinaria en funcionamiento en el sector era estadunidense (véase cuadro 3). La penetración de la USMC en Latinoamérica se inició más tarde en comparación con los grandes países industrializados europeos, pero unos años antes que en los países del sur de Europa y, como en estos últimos, la multinacional estadunidense consiguió en poco tiempo un control mayoritario del mercado (Miranda, 2004, pp. 204-205).

\section{ESTADOS UNIDOS CONTRA LA USMC}

La tendencia a la creación de grandes corporaciones impulsada por la segunda revolución tecnológica llevó al Congreso de Estados Unidos a aprobar en 1890 la Ley Sherman para proteger la competencia en el mercado al sancionar las prácticas monopolísticas y la colusión entre empresas. El objetivo no era debilitar el proceso de concentración empresarial, que se consideraba fundamental para ganar competitividad a escala mundial, sino prohibir todas aquellas prácticas que las grandes corporaciones podían utilizar para eliminar la competencia (Clark, 1900; Clark y Clark, 1912). 


\section{CUADRO 3. ORIGEN DE LAS IMPORTACIONES CHILENAS DE MAQUINARIA PARA CALZADO (VALOR EN DÓLARES DE ESTADOS UNIDOS Y PORCENTAJES), 1908-1916}

\begin{tabular}{lrrrrrr}
\hline & \multicolumn{2}{c}{1908} & \multicolumn{2}{c}{1913} & \multicolumn{2}{c}{1916} \\
Origen & Valor & Porcentaje & Valor & Porcentaje & Valor & Porcentaje \\
& & & & & & \\
Alemania & 8434 & 36.2 & 2308 & 7.6 & 698 & 4.8 \\
Estados Unidos & 6431 & 27.6 & 21715 & 71.9 & 10547 & 72.2 \\
Francia & 4977 & 21.4 & 2794 & 9.3 & 2036 & 13.9 \\
Reino Unido & 2195 & 9.4 & 2071 & 6.9 & 1061 & 7.3 \\
Otros países & 1261 & 5.4 & 1311 & 4.3 & 266 & 1.8 \\
Total & 23298 & 100 & 30199 & 100 & 14608 & 100 \\
\hline
\end{tabular}

Fuentes: elaboración propia con base en Brock (1918, p. 79), y Butman (1910a, p. 36).

Sin embargo, la ley dejó en manos de los jueces un amplio poder discrecional para establecer qué comportamientos empresariales podían considerarse ilegales e inicialmente se tendió a realizar una aplicación moderada de esta normativa, permitiendo incluso las fusiones de empresas que generaban situaciones cercanas al monopolio (Kovacic y Shapiro, 2000). De ello se benefició la USMC cuando, en 1911, el gobierno estadunidense interpuso una demanda civil en contra ante el tribunal de justicia de Massachusetts por restringir la competencia en el mercado de maquinaria para calzado. Por un lado, el gobierno acusaba a la compañía de buscar una posición de monopolio por medio de la fusión de empresas con la que se había formado y a través de las adquisiciones que había realizado posteriormente. Por otro lado, la acusaba de realizar prácticas monopolísticas mediante su sistema de arrendamiento de maquinaria y especialmente a través de algunas cláusulas de sus contratos de arrendamiento que disuadían a los clientes de utilizar maquinaria de empresas rivales. ${ }^{1}$

El tribunal del distrito falló a favor de la USMC y, tras la apelación del gobierno, esta decisión fue ratificada por el Tribunal Supremo. Los jueces dictaminaron que la formación de la compañía no había sido un intento de monopolizar el mercado, sino de reducir los costes y mejorar la eficiencia, ya que las empresas que se fusionaron en realidad no competían entre sí, sino que estaban especializadas en maquinaria de distinto tipo y, por

${ }^{1}$ United States vs. United Shoe Machinery Company of New Jersey, 247 U. S. 32 (1918). Recuperado de Public Resource Org., https://bulk.resource.org/courts.gov/c/US/247/247. US.32.207.html 
lo tanto, realizaban productos complementarios. En su opinión, tanto la fusión inicial como las adquisiciones posteriores respondían a la necesidad de la compañía de ser competitiva en un entorno tecnológico dinámico, mientras que el gran tamaño alcanzado por la empresa era consecuencia y al mismo tiempo causa de su eficiencia (Blair y Herndon, 2004, pp. 347-349). Por lo que respecta al sistema de arrendamiento, los jueces tampoco consideraron que atentase contra la libre competencia. Apoyaron su decisión en que era una estrategia que no había iniciado la USMC, sino que ya la utilizaban anteriormente las empresas que la habían constituido, y en que los clientes de la USMC eran libres de aceptar el arrendamiento, con sus condiciones, que ofrecía esta compañía o adquirir la maquinaria a otras empresas. Es más, señalaron que el sistema tenía efectos beneficiosos para la industria ya que facilitaba la entrada de nuevas empresas fabricantes de calzado, al disminuir el capital necesario, y proporcionaba un excelente sistema de mantenimiento y reparación de la maquinaria.

Ante la actitud permisiva del Tribunal Supremo a la hora de aplicar la Ley Sherman, el Congreso estadunidense decidió intensificar la política antimonopolio mediante la promulgación de las leyes Clayton y Federal Trade Commission en 1914. La Ley Clayton reducía el grado de discrecionalidad de los tribunales al prohibir específicamente, en su sección 3, ciertas prácticas, como las cláusulas en los contratos de venta o arrendamiento tendentes a reducir la competencia o los acuerdos de relación en exclusiva. Aunque este fortalecimiento de la defensa de la competencia pronto daría paso a un largo periodo de relativa tolerancia con la cooperación entre empresas y con los casos de monopolio (Kovacic y Shapiro, 2000, pp. 46-49), el gobierno estadunidense inició un nuevo proceso en 1915 contra la USMC. ${ }^{2}$ La nueva acusación era que los contratos de arrendamiento de la compañía violaban la sección 3 de la Ley Clayton y en este caso el tribunal sí falló a favor del gobierno, prohibiendo muchas de las cláusulas que obligaban a las empresas de calzado a utilizar únicamente maquinaria, accesorios o materiales de la compañía arrendadora (Blair y Herndon, 2004, pp. 350-351). La sentencia, apelada por la USMC, fue ratificada por el Tribunal Supremo, por lo que la compañía tuvo que reunirse con representantes de la National Shoe Manufacturers Association y acordar con ellos la duración y las condiciones de los nuevos contratos de arrendamiento. Estos nuevos contratos comenzaron a aplicarse a partir de noviembre de 1922. Su duración era menor, habitualmente de diez años, no incluían cláusulas que obligasen al uso de otra maquinaria de la compañía y diferenciaban los pagos por suministro de materiales de los pagos por maquinaria.

\footnotetext{
${ }^{2}$ United Shoe Machinery Corporation vs. United States, 258 U.S. 451 (1922). Recuperado de Justia, http://supreme.justia.com/cases/federal/us/258/451/case.html
} 
La política antimonopolio de la administración estadunidense volvió a endurecerse a partir de 1936, a la vez que decaía la confianza en los modelos de planificación económica y el presidente Roosevelt se dejaba aconsejar por quienes consideraban que impulsar la competencia era la llave para la recuperación económica. La nueva campaña contra la colusión entre empresas y los monopolios se vio fortalecida por los trabajos de los economistas de la Universidad de Chicago Jacob Viner, Frank Knight y, particularmente, Henry Simons, quienes defendieron el libre mercado, la competencia y las medidas antimonopolio frente al intervencionismo gubernamental. Durante las décadas de 1940, 1950 y 1960, los tribunales aumentaron el control sobre los acuerdos de colaboración entre empresas y sobre las compañías con una posición dominante en el mercado (Kovacic, 1992).

En este contexto, era inevitable que se volviese a cuestionar la situación de la USMC. En efecto, el gobierno federal acusó de nuevo a la compañía, ante el tribunal del distrito de Massachusetts, de haber violado continuamente desde 1912 las secciones 1 y 2 de la Ley Sherman al monopolizar la fabricación y distribución de maquinaria para calzado y para la industria del curtido, el suministro de repuestos y accesorios para dicha maquinaria y el abastecimiento de materiales para la fabricación de calzado. Al igual que los litigios anteriores, el proceso, iniciado en 1947, se prolongó durante varios años, pero finalmente en febrero de 1953 el tribunal de Massachusetts falló en contra de la USMC y dictaminó que esta compañía efectivamente monopolizaba el mercado de maquinaria para calzado y de algunos suministros para esta industria. ${ }^{3}$

Según la sentencia, el dominio sobre el mercado que ejercía la compañía no sólo se debía a su eficiencia y a la fortaleza obtenida con la fusión inicial de las principales empresas de su sector, cuya legalidad había sido ratificada en 1918 por el Tribunal Supremo, sino también al empleo de ciertas prácticas empresariales que habrían actuado como barreras a la competencia. Entre estas prácticas se destacaba la adquisición de patentes, el control de la maquinaria de segunda mano $y$, muy especialmente, el sistema de arrendamiento. El Tribunal Supremo avaló la sentencia de la Corte de Massachusetts en mayo de 1954 y aunque no se aceptó la propuesta del gobierno de fragmentar la compañía, sí se obligó a esta a modificar sustancialmente su estrategia y a disminuir sus activos (Kaysen, 1956, pp. 382-388).

Concretamente, la sentencia obligaba a la USMC a poner también a la venta cualquier modelo de máquina que se ofreciese en arrendamiento

${ }^{3}$ United States vs. United Shoe Machinery Company, 110 F. Supp. 295 (1953). Recuperado de Court Listener, https://www.courtlistener.com/mad/aCn4/united-states-v-united-shoe-machinerycorp/ 
y a que las condiciones de ambas posibilidades debían ser tales que no resultase sustancialmente más ventajoso arrendar que comprar la maquinaria. Los nuevos contratos de arrendamiento no debían durar más de cinco años y el arrendatario podía cancelarlos a voluntad una vez que hubiese transcurrido el primer año. No debía existir ninguna penalización por devolver la maquinaria para sustituirla por otra de la competencia. Tampoco podían establecerse niveles mensuales mínimos en los pagos por grado de utilización de las máquinas, ni cláusulas que obligasen a utilizar el máximo de capacidad de la maquinaria. Además, el servicio de asistencia técnica debía ser cobrado por separado, al igual que el desplazamiento de su personal. Se encargaba a la USMC preparar un plan, antes del 1 de abril de 1955, para poner fin a los contratos de arrendamiento en vigor y reemplazarlos por ventas o contratos de arrendamiento ajustados a los términos de la sentencia.

Este plan había de acordarse con el gobierno y con representantes de la National Shoe Manufacturers Association. La compañía y sus empresas filiales debían deshacerse de todo su negocio de fabricación y distribución de tachuelas, clavos y ojetes, y debían cesar de distribuir materiales no fabricados por ellas. También se prohibía a la USMC adquirir patentes, a menos que aceptase dar licencia a otras empresas para el uso de esas patentes a un precio razonable; la compañía estaba obligada a conceder, a cualquiera que lo solicitase, una licencia no exclusiva sobre las patentes que poseía hasta el momento de la sentencia. Aunque la decisión del tribunal sólo afectaba las actividades de la compañía en Estados Unidos, los cambios que originó en la estrategia de la USMC acabaron trasladándose también a sus filiales en el extranjero (Howie, 1999, p. 93).

La sentencia ordenaba que, transcurridos diez años, ambas partes realizasen una valoración de los resultados de las medidas adoptadas para dictaminar si era necesario modificarlas. Por ello, en 1965 el gobierno presentó un informe en el que exponía que la USMC había mantenido un elevado control sobre el mercado de maquinaria para calzado y solicitaba, de nuevo, la división de la corporación. La USMC, por el contrario, argumentó que las medidas habían cumplido su objetivo de disminuir la participación de la empresa en el sector, que se había reducido en casi un tercio y, por ello, solicitaba que se redujesen las restricciones impuestas. El juez Wyzanski, encargado del caso en el tribunal del distrito de Massachusetts, se mostró satisfecho con los resultados del decreto de 1953 y no modificó sus disposiciones. ${ }^{4}$ Sin embargo, el gobierno apeló al Tribunal

\footnotetext{
${ }^{4}$ United States vs. United Shoe Machinery Corporation, 266 F. Supp. 328 (1967). Recuperado de Court Listener, https://www.courtlistener.com/mad/9BXN/united-states-v-uni ted-shoe-machinery-corporation
} 
Supremo y obtuvo el respaldo de este para exigir medidas más radicales contra la posición dominante de la USMC. Como resultado, el tribunal de Massachusetts decretó en 1969 que la compañía debía desprenderse de al menos un tercio de sus activos. ${ }^{5}$

Ante la necesidad de reducir su presencia en el mercado de maquinaria para calzado, la compañía adoptó desde mediados de la década de 1950 y especialmente durante la de 1960, la estrategia de diversificar sus actividades e invertir en otras producciones. Para ello adquirió más de 40 empresas, en Estados Unidos y en otros países, entre 1962 y 1973. Sin embargo, muchos de los proyectos de diversificación no resultaron rentables, el valor de las acciones de la compañía se redujo drásticamente y ello permitió que en 1976 fuese adquirida por una firma de Nueva Inglaterra, especializada en maquinaria para la industria del vidrio, que tenía la mitad de su tamaño. Dejó así de ser una empresa independiente para convertirse en una división de la Emhart Corporation.

En los años siguientes, la USMC continuó liderando a escala mundial la industria de maquinaria para calzado, pero con una cuota de mercado en rápida disminución. Su trayectoria se vio perjudicada por tres factores. Por un lado, los cambios de localización de la industria del calzado a escala global, con el descenso acelerado de la producción en Estados Unidos y la expansión exportadora de esta industria en el sur de Europa y Extremo Oriente, que fomentó el desarrollo en dichas zonas, especialmente en Italia, de industrias de maquinaria para calzado muy competitivas en precio. Por otro, el profundo cambio tecnológico que afectó al sector con la introducción de los sistemas hidráulicos y neumáticos, los sistemas de control numérico y posteriormente de la electrónica, que hizo perder a la USMC buena parte de su ventaja tecnológica y facilitó la entrada de nuevos competidores en el sector. Finalmente, la gestión poco afortunada dentro de un grupo industrial con intereses diversos. Esta última razón conduciría a que la compañía trasladase su centro de dirección a Europa, ya que Emhart formuló a mediados de la década de 1980 una estrategia en la que no había lugar para las industrias intensivas en capital sujetas a fluctuaciones cíclicas.

Como respuesta, un grupo de directivos de la propia compañía compró, en 1987, la parte dedicada a la maquinaria por 80000000 de libras esterlinas y fundó el United Machinery Group (UMG), con centros de producción en Estados Unidos, Alemania, Brasil, Taiwán y Reino Unido, y sede central en este último país (Howie, 1999, pp. 99-118).

${ }^{5}$ United States vs. United Shoe Machinery Corporation, 391 U.S. 244 (1969). Recuperado de Justia, http://supreme.justia.com/cases/federal/us/391/244/case.html 


\section{¿BÚSQUEDA DEL MONOPOLIO O ESTRATEGIA EMPRESARIAL EFICIENTE?}

Recientemente, diversos autores han revisado el caso de la USMC y han llegado a la conclusión de que las prácticas de la compañía que los tribunales consideraron que atentaban contra la competencia, principalmente el sistema de arrendamiento, en realidad no lo hacían y, en cualquier caso, formaban parte de una estrategia empresarial eficiente, que se vio deteriorada por las medidas antimonopolio (Blair y Herndon, 2000, 2004; Crandall y Elzinga, 2004; Masten y Snyder, 1999; Wiley, Rasmusen y Ramseyer, 1990). Masten y Snyder (1999), por ejemplo, defienden que el sistema de arrendamiento era una buena vía para asegurar la calidad de la maquinaria y para proporcionar los servicios y la información que requería el uso de dicho equipamiento. En su opinión, la larga duración de los contratos y las cláusulas que fueron prohibidas por los tribunales tendían, en efecto, a impedir que los fabricantes de calzado adquiriesen maquinaria de la competencia, pero este efecto negativo se veía compensado porque permitían abaratar los costes asociados a la relación comercial entre el fabricante de maquinaria y sus clientes. Desde este enfoque, tanto la duración como las cláusulas restrictivas de los contratos establecerían incentivos adecuados para el uso de la maquinaria y, sobre todo, para evitar que los arrendatarios pudiesen eludir un pago suficiente por la información y los servicios recibidos de la United Shoe Machinery Company.

Prueba de que el arrendamiento era una solución a los problemas de organización del sector fue que otras empresas de maquinaria para calzado, antes y después de la constitución de la USMC, también habían utilizado este sistema con parecidas condiciones. $\mathrm{El}$ arrendamiento transformaba la transacción entre la USMC y las empresas de calzado; esta pasaba de ser la venta discreta de un bien duradero a ser la venta continuada de servicios vinculados con la maquinaria. Desde el punto de vista de la economía funcional, este fenómeno ya sería en sí mismo positivo, puesto que contribuiría a optimizar el uso de los recursos (Stahel, 2005). Sin embargo, lo que destacan Masden y Snyder (1999) es que esta transformación aportaba dos ventajas fundamentales: incentivaba a la USMC a desarrollar maquinaria compleja y a mantenerla en perfecto estado, y facilitaba la transferencia de asesoramiento técnico a los fabricantes de calzado.

Blair y Herndon (2000) destacan otras ventajas del sistema de arrendamiento, como su capacidad de disminuir las exigencias financieras a las empresas de calzado, bajando las barreras de entrada en el sector, y la protección que proporcionaba al arrendatario del riesgo de que la máquina no tuviese un buen rendimiento, exigiese un mantenimiento costoso o quedase obsoleta. Respecto a la estrategia de la compañía de no cobrar separadamente por el servicio técnico, tampoco consideran que fuese una 
práctica monopolística, sino que era una estrategia históricamente utilizada en la industria, que reducía el riesgo de sufrir problemas técnicos y costes asociados a ellos para los fabricantes de calzado y aseguraba el mantenimiento de sus equipos al arrendador. Era, además, una estrategia socialmente eficiente, ya que, al disminuir el riesgo, los fabricantes de calzado incrementaban su demanda de maquinaria, aumentaban su productividad y producían más calzado a menores precios.

Ambos autores intentan demostrar también que ni el arrendamiento en sí mismo ni las cláusulas utilizadas por la USMC tenían un efecto anticompetitivo. En este sentido coinciden con Wiley, Rasmusen y Ramseyer (1990) en que la adopción del sistema de arrendamiento por parte de la USMC no era una forma de mantener los precios de monopolio en el tiempo, es decir, no era una respuesta a lo que actualmente se conoce como la conjetura de Coase. Esta teoría, desarrollada por Ronald Coase (1972), establece que cuando un monopolista produce bienes duraderos, que pueden venderse a lo largo del tiempo, acaba compitiendo consigo mismo, ya que los clientes anticipan futuras reducciones de precios y pueden demorar su compra, con lo que la empresa monopolista no podrá mantener un precio de monopolio y deberá establecer un precio competitivo. Una vía que tiene la empresa para evitar este problema es arrendar sus productos en lugar de venderlos, siempre que el plazo de arrendamiento sea suficientemente corto. Sin embargo, no puede considerarse que la USMC buscase en el arrendamiento una forma de evitar la conjetura de Coase porque sus contratos de arrendamiento no eran de corto plazo. Además, la teoría de Coase parte del supuesto de que el número de compradores es fijo, mientras que el mercado de la maquinaria para el calzado estaba en continua renovación por la entrada de nuevas empresas en el sector (Blair y Herndon, 2004, pp. 365-366).

\section{LAS CONSECUENCIAS DEL MONOPOLIO \\ Y DE LAS MEDIDAS ANTIMONOPOLIO}

Frente a lo que pronosticaría la teoría económica neoclásica, no hay evidencias sólidas de que la posición dominante en el mercado de la USMC originase un aumento de los precios de la maquinaria ni una reducción de su oferta. Según Kaysen (1956), los beneficios netos de la compañía después de impuestos eran alrededor de 10\% del capital invertido, es decir, similares a los obtenidos por otras empresas en industrias de características parecidas e inferiores a los de otras grandes corporaciones. En realidad, su principal empresa rival, Compo, que controlaba un porcentaje del mercado muy inferior, tenía una tasa de beneficios más alta. Esto parece indicar 
que los precios de la USMC no debían estar muy por encima de los precios de mercado en competencia y lo mismo ocurriría en las empresas filiales de la USMC en otros países; como lo prueba un informe elaborado en 1919 por una comisión nombrada por el gobierno británico, que indicaba que no había evidencias de que el predominio de la British United Shoe Machinery Company ocasionase mayores costes para los fabricantes de calzado (British Boot Machinery Manufacturers' Association, c. 1919, pp. 73-74). De hecho, las tarifas por el uso de la maquinaria era la única partida de los costes de producción del calzado en Estados Unidos que resultaba menor una década después que en 1899, cuando se creó la United Shoe Machinery Company.

Además, el coste del arrendamiento de la maquinaria por unidad de producto fue disminuyendo con el tiempo. Si en 1911 el cálculo del coste medio del arrendamiento de la maquinaria por par de zapatos era de 0.26 dólares aproximadamente ( 0.13 dólares si se exceptuaba el calzado empalmillado, que sólo era un tercio de la producción total) (United Shoe Machinery Company, 1912, p. 39), a principios de la década de 1950 este coste medio se redujo entre 0.07 y 0.10 dólares por par (Kaysen, 1956, pp. 314-329). Hubo quejas de fabricantes de calzado contra las tarifas de la USMC, que se consideraban excesivamente elevadas. Sin embargo, los datos disponibles no sugieren que el coste del arrendamiento de la maquinaria resultase particularmente gravoso, ni en Estados Unidos ni en los otros países. Así, por ejemplo, en una empresa de calzado de Turín, las regalías pagadas a la USMC en 1913 no representaban más que 3.5\% de los costes de producción de un par de zapatos de hombre (Butman, 1913b, p. 8).

Las asociaciones de productores de calzado protestaron principalmente contra las cláusulas de los contratos de arrendamiento de la USMC que limitaban la libertad de elección de los fabricantes de calzado y, sobre todo, la posibilidad de utilizar maquinaria de la competencia. Hubo quejas en este sentido, por ejemplo de la Boot and Shoe Manufacturers' National Association de Estados Unidos, la Western Shoe Manufacturers' Association, la Brockton Shoe Manufacturers' Association, la London Boot and Shoe Manufacturers' Association y la asamblea de presidentes de las cámaras de comercio francesas (British Boot Machinery Manufacturers' Association, c. 1919, p. 71; Le Bot, 2007, p. 117; Weekly Bulletin of Leather and Shoe News, 1911, pp. 69-85). No obstante, estas reivindicaciones fueron promovidas probablemente por las empresas de mayores dimensiones, que eran las que menos se beneficiaban del sistema de asistencia técnica y de las facilidades de acceso a la maquinaria que proporcionaba la USMC, mientras que los pequeños empresarios tendrían una actitud más favorable a la multinacional. Por ello, en una encuesta efectuada en 1911 por un diario estadunidense, el Boston News Bureau, sobre la satisfacción de los fabrican- 
tes de calzado con el servicio de la USMC, casi las dos terceras partes de las más de 400 respuestas recibidas fueron positivas, y los fabricantes de calzado que declararon en el proceso contra la USMC abierto en 1947 también aseguraron sentirse bien servidos por la compañía (Masten y Snyder, 1999, p. 41; Weekly Bulletin of Leather and Shoe News, 1911, p. 115).

En cualquier caso, lo que nunca se cuestionó fue la calidad de la maquinaria ni del servicio técnico de la USMC, tanto en Estados Unidos como en los otros países donde operaban filiales de la multinacional. Incluso las otras empresas de maquinaria para calzado establecidas en el Reino Unido, en su informe contra la posición monopolística de la British United Shoe Machinery Company en su país, reconocían en 1918 que esta "es una compañía eficiente", "proporciona un buen servicio a sus clientes" y "ha prestado indudablemente un buen servicio a la industria del calzado de Gran Bretaña”. Lo mismo sostenía el informe anteriormente citado de la Comisión británica de expertos, para la que "la eficiencia de la organización de la empresa y la calidad de su maquinaria eran incuestionables" (British Boot Machinery Manufacturers' Association, c. 1919, pp. 40 y 73).

La teoría de los mercados disputados (contestable markets) proporciona una explicación coherente de por qué la USMC no aprovechó su posición dominante para imponer precios más altos y conseguir mayores beneficios o disminuir la calidad de su servicio. Según esta teoría, desarrollada por Baumol, Panzar y Willing (1982), cuando en una situación de monopolio u oligopolio existen competidores potenciales que podrían entrar en el mercado y reducir la participación de las empresas dominantes, estas se ven obligadas a actuar de forma competitiva, tanto mediante el control de sus precios como a través de la innovación y la mejora de sus productos y servicios. No obstante, para que actúe este mecanismo es necesario que nuevos competidores puedan entrar y salir fácilmente del mercado y, por lo tanto, que no existan costes hundidos importantes. Existen planteamientos similares a principios del siglo Xx, por ejemplo, John Bates Clark (1900), para quien "the new mills that will spring into existence will break down prices; and the fear of these new mills, without their actual coming, is often enough to keep prices from rising to an extortionate height. The mill that has never been built is already a power in the market; for if it surely will be built under certain conditions, the effect of this certainty is to keep prices down" (p. 50).

Las características de la industria de maquinaria para calzado en la primera mitad del siglo XX se ajustan básicamente a las definidas para los mercados disputados. La USMC tenía una posición claramente dominante, en Estados Unidos y en muchos otros países, pero también existía un buen número de empresas mucho más pequeñas, especializadas en ciertos tipos de maquinaria, que podían aprovechar cualquier comportamien- 
to deficiente de la compañía dominante, en forma de precios elevados, mala calidad del producto o inadecuación de este a las necesidades de las empresas de calzado, para lanzar un producto alternativo y reducir la cuota de mercado de la USMC. A mediados de siglo, había en Estados Unidos 22 empresas que fabricaban algunas de las máquinas consideradas fundamentales en la industria del calzado y 83 que fabricaban maquinaria auxiliar. La mayor de esas empresas era Compo, cuyos activos equivalían a menos de $4 \%$ de los activos de la USMC. Se trataba, por lo tanto, de muy pequeños competidores pero que contaban con información sobre el mercado y sobre la tecnología del sector, y con una estructura productiva y comercial que les permitía detectar las oportunidades para entrar en el mercado de un determinado tipo de maquinaria en cualquier momento. Un buen ejemplo del potencial competitivo de estas empresas lo proporciona Compo, que supo apreciar las posibilidades de la maquinaria para el pegado de la suela antes que la USMC y logró hacerse con la mitad del mercado para esta tecnología en Estados Unidos (Kaysen, 1956, pp. 52-53 y 366-369).

La USMC controlaba una gran parte de las patentes de maquinaria para el calzado registradas en Estados Unidos y este control de la tecnología probablemente pudo actuar como una decisiva barrera de entrada en la industria en las primeras décadas del siglo, pero no parece que continuase así tras la segunda guerra mundial, ya que todas las patentes importantes habían pasado a dominio público y podían ser copiadas libremente. Tampoco parece que existiesen economías de escala importantes en la producción de maquinaria específica, que actuasen como barreras de entrada en el sector, ya que las series de producción de cada máquina eran cortas y los distintos modelos de maquinaria compartían un número reducido de piezas comunes. Por lo tanto, la existencia de potenciales competidores habría obligado a la compañía a seguir con atención la situación del mercado, como si se hubiese tratado de una estructura industrial menos concentrada. De hecho, existe constancia documental de que la USMC estaba muy atenta al comportamiento de las otras empresas de maquinaria y sus servicios técnicos elaboraban continuamente informes sobre la presencia de productos de la competencia en las fábricas de sus clientes (Masten y Snyder, 1999, p. 40).

Lo que sí es seguro es que las medidas antimonopolio aplicadas a la compañía a partir de 1954 no originaron un descenso de los precios, sino justo lo contrario. Los precios de venta de las máquinas se multiplicaron por cuatro como media y en los nuevos contratos de arrendamiento, aunque las rentas por unidad de uso de la maquinaria descendieron alrededor de $25 \%$, las rentas fijas mensuales aumentaron en muchas ocasiones más de 500\%. De acuerdo con los cálculos efectuados por la National Shoe 
Manufacturers Association, los nuevos contratos de puestos en vigor a partir del 1 de enero de 1955 incrementaban el coste del arrendamiento del conjunto de la maquinaria en $74 \%$ para las empresas pequeñas (con una producción inferior a 1800 pares al día), en 65\% para las mediadas (de 1800 a 3600 pares) y en 57\% para las grandes (más de 3600 pares) (Masten y Snyder, 1999, p. 329).

Para observar el efecto de la sentencia contra la USMC sobre los precios de la maquinaria para calzado en Estados Unidos, Crandall y Elzinga (2004) han utilizado como indicador indirecto la ratio "valor de la maquinaria comercializada/valor de las ventas de calzado". Su razonamiento es que si la sentencia hizo que se redujesen los precios de la maquinaria, los fabricantes de calzado se habrían encontrado con un menor gasto en maquinaria con relación al valor del calzado producido; por lo tanto, la ratio tendría que haber descendido. Sin embargo, la ratio, que sí se había reducido en los años previos a la sentencia, se incrementó después de ella y hasta principios de la década de 1970. Por lo tanto, las medidas antimonopolio sobre la USMC no consiguieron que esta compañía redujese sus precios ni tampoco un aumento de la competencia que se reflejase en un descenso general de los precios de la maquinaria para calzado.

Masten y Snyder (1999) consideran que la sentencia tuvo, además, varios efectos perjudiciales. Por un lado, la obligación de tener que ofrecer en venta toda la maquinaria en condiciones tan favorables como las del arrendamiento, junto a la separación del servicio técnico, redujo las ventajas proporcionadas por el anterior sistema para impulsar el esfuerzo en investigación de la USMC, trasladar información a sus clientes y garantizar la calidad de la maquinaria y su mantenimiento. Como indicador de esto último, utilizan el número de técnicos itinerantes de la empresa en Estados Unidos, que eran fundamentales para reparar la maquinaria y proporcionar un asesoramiento continuo a los clientes. Este número pasó de 846 en 1955 (6.4 por cada 1000 máquinas de la USMC en funcionamiento) a tan sólo 349 (3.7) en 1964. Por otro lado, la medida citada también limitó las posibilidades de la compañía de evitar los comportamientos oportunistas en sus clientes, lo que se vio agravado por la prohibición en los nuevos contratos de arrendamiento de las cláusulas de uso mínimo y de utilización a plena capacidad (pp. 65-66).

Diversos autores sugieren que las medidas contra la estrategia empresarial tradicional de la USMC contribuyeron al declive de la industria del calzado en Estados Unidos desde finales de la década de 1950. Sin embargo, la involución de esta industria obedeció fundamentalmente a otras razones. Fue la competencia del calzado importado, procedente de países con costes salariales menores, y la actitud de los distribuidores y de los propios productores estadunidenses, que prefirieron subcontratar la pro- 
ducción e incluso establecer factorías propias en estos países, la principal razón del declive del sector en Estados Unidos.

Si la situación de la USMC acentuó este proceso fue de forma muy leve y a través del incremento del coste de la maquinaria que se produjo tras la sentencia de 1953, que se habría reflejado en un ligero aumento de los costes de producción en Estados Unidos. En realidad, la causalidad actuó en sentido inverso. Fue el retroceso de la industria del calzado en Estados Unidos lo que actuó como una influyente causa del declive de la USMC. Sin duda, las restricciones impuestas por los tribunales contribuyeron a la pérdida de competitividad de la compañía y a la reducción de su volumen de negocio, pero ambos fenómenos también se hubiesen dado aunque la USMC no hubiese sufrido las medidas antimonopolio. Difícilmente la compañía hubiese podido mantener su predominio en el mercado internacional desde Estados Unidos sin el apoyo de una amplia demanda interna. El debilitamiento de esta demanda agravó los efectos del cambio tecnológico y la consiguiente pérdida de la ventaja competitiva que la USMC había disfrutado durante décadas por su dominio de las patentes y del know-how en el tipo de sistemas mecánicos que predominaron en la industria del calzado hasta la década de 1950.

\section{CONCLusiones}

La USMC disfrutó de una posición muy próxima al monopolio en el mercado de maquinaria para calzado de Estados Unidos y otros países durante la primera mitad del siglo Xx. Sin embargo, no parece que ese poder de monopolio se reflejase en una elevación de los precios y una disminución de la oferta de maquinaria, probablemente porque se trataba de un mercado disputado en el que la posibilidad de que interviniesen potenciales competidores incentivaba a la compañía a proporcionar una oferta eficiente, con calidades y precios competitivos.

El sistema de arrendamiento, las cláusulas restrictivas del mismo y la inclusión en él del servicio técnico colaboraron a mantener el predominio de la USMC, pero no fueron sus causas fundamentales. Estas prácticas, que acabaron siendo limitadas y hasta prohibidas por los tribunales, colaboraban eficazmente con el desarrollo de la actividad de la compañía y no eran medidas adoptadas exclusivamente por la USMC, sino que también habían sido y eran utilizadas por otras empresas del sector.

El verdadero pilar de la posición dominante de la USMC era su superioridad tecnológica. Una superioridad conseguida por la acumulación de patentes y del know-how desde el mismo momento de su constitución, con la fusión de las principales empresas del sector, y que fue alimentada pos- 
teriormente con fuertes inversiones en investigación y, en menor medida, con la compra de patentes e invenciones. El predominio de la compañía también se apoyó en las importantes economías de alcance de que disfrutaba, por las sinergias existentes entre la producción de los distintos tipos de maquinaria, el servicio técnico y la producción y distribución de accesorios, componentes y materiales para el calzado. Otro factor de éxito nada desdeñable fue la buena gestión de la empresa.

La causa fundamental del declive de la USMC fue precisamente la pérdida de su superioridad tecnológica por el desarrollo de nuevos sistemas para este tipo de maquinaria, pérdida reforzada por el fuerte retroceso de la industria del calzado en Estados Unidos y en el norte de Europa, que eran los principales mercados tradicionales para la compañía. También colaboraron al declive las medidas judiciales, particularmente por la pérdida de parte de las economías de alcance anteriormente señaladas, pero sin ellas se hubiese erosionado igualmente el predominio de la USMC. $\dot{¿}$ Consiguieron estas medidas reducir el poder de mercado de la USMC en Estados Unidos e impulsar la competencia? Evidentemente, la cuota de mercado de la USMC disminuyó desde mediados de la década de 1950 a favor de otras empresas estadunidenses y de la maquinaria importada. Sin embargo, no parece que mejorase la oferta en el sentido de que hubiese una disminución del precio (al contrario, los precios se incrementaron) o una mejora de la calidad y las prestaciones de las máquinas. El cambio tecnológico que afectó a la maquinaria para calzado a partir de la década de 1960 no se dio porque disminuyese el predominio de la USMC, sino por la propia evolución de la tecnología.

Aunque algunas posiciones teóricas consideran que cualquier limitación de la competencia genera ineficiencia, la trayectoria de la USMC demuestra que una empresa con una posición dominante en el mercado puede tener un desempeño eficiente. De hecho, con la USMC la modernización de la industria del calzado progresó mucho más rápidamente, sobre todo fuera de Estados Unidos, que antes de la constitución de esta compañía, cuando había una multitud de empresas compitiendo en el sector de maquinaria para el calzado. El sistema de arrendamiento de la USMC rebajó las barreras de entrada en el sector y, junto a su excelente servicio técnico, redujo la incertidumbre de las fábricas de calzado sobre la inversión en tecnología. La fortaleza de la compañía le permitió trasladar su estrategia empresarial a multitud de países y, así, impulsar en ellos la misma modernización que había relanzado en Estados Unidos. Su departamento de I+D generó numerosas innovaciones, que favorecieron decisivamente la mejora de la productividad, y sus precios tendieron a ser competitivos. De esta forma, aunque la USMC redujo la competencia en el mercado de maquinaria para calzado, contribuyó decisivamente al crecimiento de la 
competencia en la industria del calzado, al facilitar el establecimiento de nuevas empresas bien equipadas.

La experiencia de los años previos a la constitución de la USMC parece indicar que se habría conseguido un nivel similar de progreso tecnológico con una industria de maquinaria menos concentrada empresarialmente. Incluso quizá se podría haber disfrutado de una red de servicio técnico igual de extensa y eficaz en Estados Unidos. No obstante, fue la concentración de recursos conseguida por la USMC lo que hizo posible la rápida difusión de la tecnología estadunidense para calzado en otros países desde principios del siglo XX. El predominio de la maquinaria estadunidense impidió el desarrollo de una producción importante de maquinaria en la primera mitad del siglo en muchos países, pero hizo posible en cambio que esos mismos países modernizasen su industria del calzado. Las empresas de calzado estadunidense penetraron con mucho éxito en el mercado internacional desde finales del siglo XIX, gracias a su mejor equipamiento, llegando a convertir a Estados Unidos en el mayor exportador mundial de calzado a principios del siglo Xx. Sin embargo, la rápida difusión de la tecnología estadunidense en el resto del mundo, gracias a la USMC, permitió a muchos países competir eficazmente con estas importaciones y, en algunos casos, convertirse incluso en destacados exportadores a Estados Unidos.

\section{LISTA DE REFERENCIAS}

BARBERo, M. I. (2011). Construyendo activos intangibles. La experiencia de una empresa fabricante de calzado en la Argentina de la primera mitad del siglo Xx. Revista de la Historia de la Economía y de la Empresa, 5, 151-176.

Baumol, W. J., Panzar, J. C. y Willig, R. D. (1982). Contestable markets and the theory of industry Structure. Orlando: Harcourt Brace Jovanovich.

Bibiloni, A. y Pons, J. (2001). El lento cambio organizativo en la industria del calzado mallorquina (1900-1960). En C. ARENAS, A. Florencio y J. Pons (eds.), Trabajo y relaciones laborales en la España contemporánea (pp. 355-370). Sevilla: Mergablum.

Blair, R. D. y HERndon, J. B. (2000). United Shoe Machinery and the antitrust significance of 'free' Service. Review of Industrial Organization, 17, 301-311.

BlaiR, R. D. y Herndon, J. B. (2004). United Shoe Machinery revisited. Research in Law and Economics, 21, 347-349.

British Boot Machinery Manufacturers' Association (c. 1919). The growth of a monopoly: The history of the shoe machinery monopoly, 1899-1918. Leicester: British Boot Machinery Manufacturers' Association.

BRock, H. G. (1918). Markets for boots and shoes in Chile and Bolivia. Washington: Department of Commerce. 
Brock, H. G. (1919) Boots and shoes, leather, and supplies in Argentina, Uruguay and Paraguay. Washington: Department of Commerce.

Butman, A. B. (1909). Shoe and leather trade in Cuba and Mexico. Washington: Department of Commerce and Labor.

Butman, A. B. (1910a). Shoe and leather trade in Argentina, Chile, Peru, and Uruguay. Washington: Department of Commerce and Labor.

Butman, A. B. (1910b). Shoe and leather trade in Brazil, Venezuela, and Barbados. Washington: Department of Commerce and Labor.

Butman, A. B. (1912a). Shoe and leather trade in France and Switzerland. Washington: Department of Commerce and Labor.

Butman, A. B. (1912b). Shoe and leather trade in the United Kingdom. Washington: Department of Commerce and Labor.

Butman, A. B. (1912c). Shoe and leather trade in Germany. Washington: Department of Commerce and Labor.

Butman, A. B. (1913a). Shoe and leather trade in Belgium, Spain and Egypt. Washington: Department of Commerce and Labor.

Butman, A. B. (1913b). Shoe and leather trade in Italy and Austria-Hungary. Washington: Department of Commerce and Labor.

Church, R. A. (junio, 1968). The effect of the American export invasion on the British Boot and shoe industry 1885-1914. The Journal of Economic History, 28(2), 223-254.

Clark, J. B. (enero, 1900). Disarming the trusts. The Atlantic Monthly, 85(507), 47-55.

Clark, J. B. y Clark, J. M. (1912). The control of trust. Nueva York: MacMillan.

COASE, R. (1972). Durability and monopoly. Journal of Law and Economics, 15(1), 143-49.

Crandall, R. W. y Elzinga, K. G. (2004). Injunctive relief in Sherman Act monopolization cases. Research in Law and Economics, 21, 277-344.

EsCARTín, J. M. (2001). La ciutat amuntegada. Indústria del calçat, desenvolupament urbà $i$ condicions de vida en la Palma contemporània (1840-1940). Palma: Documenta Balear.

Federal Trade Commission (1919). Report on leather and shoe industries. August 21, 1919. Washington: Government Printing Office.

Fontana, G. L. (ed.). (1998). 100 Anni di industria calzaturiera nella Riviera del Brenta. Venecia: Associazione CalzaTurifici Riviera del Brenda, ACRIB.

Hoover, E. M. (1937). Location theory and the shoe and leather industries. Cambridge: Harvard University Press.

HowIE, I. (1999). Serving the shoemaker for 100 years. The oficial centenary history of British United Shoe Machinery. Leicester: British United Shoe Machinery Co.

KABAT, M. (2001). Transformaciones en los procesos de trabajo durante los inicios de la industria del calzado en la Argentina (1880-1920). $5^{\circ}$ Congreso Nacional de Estudios del Trabajo. Asociación Argentina de Especialistas en Estudios del Trabajo, Argentina. Recuperado de http://www.aset.org.ar/congresos/5/aset/PDF/KABAT.PDF

KAYSEn, C. (1956). United States v. United Shoe Machinery Corporation. An economic analysis of anti-trust case. Cambridge: Harvard University Press. 
Kovacic, W. E. (abril, 1992). The influence of economics on antitrust law. Economic Inquiry, 30(2), 294-306.

Kovacic, W. E. y Shapiro, C. (2000). Antitrust policy: A century of economic and legal thinking. Journal of Economic Perspectives, 14(1), 43-60.

LE Bot, F. (2007). La fabrique réactionnaire. Antisémitisme, spoliations et corporatisme dans le cuir (1930-1950). París: Presses de la Fondation nationale des Sciences Politiques.

Manera, C. (ed.). (2002). Las islas del calzado. Historia económica del sector en Baleares (1200-2000). Palma de Mallorca: Lleonard Muntaner.

Masten, S. E. y Snyder, E. A. (abril, 1999). United States versus United Shoe Machinery Corporation: On the merits. Journal of Law and Economics, 36, 33-70.

Miranda, J. A. (1998). La industria del calzado en España (1860-1959). La formación de una industria moderna y los efectos del intervencionismo estatal. Alicante: Generalitat Valenciana.

Miranda, J. A. (abril, 2004). American machinery and European footwear: Technology transfer and international trade, 1860-1939. Business History, 46(2), pp. 195-218.

Miranda, J. A. (2009). Competing in fashion goods: Firms and industrial districts in the development of the Spanish shoe industry. Business and Economic History onLine, 7, 1-34.

NADAL, J. (1994). La transición del zapato manual al zapato mecánico. En J. NADAL y J. CATAlan (eds.), La cara oculta de la industrialización española. La modernización de los sectores no líderes (siglos XIX y XX) (pp. 321-339). Madrid: Alianza Universidad.

Ponce, G. y Martínez, A. (2003). La industria del calzado en el Alto Vinalopó (1850-1977). Origen y expansión de una manufactura. Murcia: Caja de Ahorros del Mediterráneo.

SABbatuCCI, P. (1998). Due distretti calzaturieri a confronto: Vigevano e i paesi del Fermano dalla metà dell'Ottocento agli anni del boom economico. Proposte e Ricerche, 40, 93-118.

Sabbatucci, P. (2001). Il distretto calzaturiero marchigiano (1910-1960): alle origini di una grande affermazione. En F. Amatori y A. Colli (eds.), Comunità di imprese. Sistemi locali in Italia tra ottocento e novecento (pp. 361-412). Bolonia: Il Mulino.

SabbatuCCI, P. (2007). Les districts de production de chaussures en Italie, 1880-1980. En J. C. Daumas, P. Lamard y L. Tissot (eds.), Les territoires de l'industrie en Europe 1750-2000 (pp. 361-382). Besanzón: Presses Universitaires de Franche-Conté.

Sabbatucci, P. (2010). Société et institutions dans le développement des districts des chaussures des Marches, 1880-1970. En L. TISsOT (ed.), Histoires de territoires. Les territoires industriels en question, XVIII-XX siècles (pp. 225-252). Neuchâtel: Alphil-Presses Universitaires Suisses.

SCHNitzer, J. G. (1937). Leather footwear. World production and international trade. Washington: US Department of Commerce.

SEgReto, L. (1989). L'industria calzaturiera in Italia. La lunga rincorsa marchigiana, 1914-1960. En S. Anselmi (ed.), L'industria calzaturiera marchigiana. Dalla manifattura alla fabbrica (pp. 247-323). Fermo: Unione Industriali del Fermano. 
STAHEL, W. R. (octubre, 2005). The functional economy: Cultural and organizational change. International Journal of Performability Engineering, 1(2), 121-130.

The Leather Trade's Review. 14 de noviembre de 1928.

Thomson, R. (1989). The path to mechanized shoe production in the United States. Chapel Hill y Londres: The University of North Carolina Press.

United Shoe Machinery Company (1912). The story of three partners. Beverly: United Shoe Machinery Co.

Valero, J. R., Navarro, A., Martínez, F. y Amat, J. M. (1992). Elda, 1832-1980 industria del calzado y transformación social. Elda: Instituto de Cultura Juan Gil-Albert/ Ayuntamiento de Elda.

Weekly Bulletin Of Leather And Shoe News (1911). Discussion of shoe machinery. The real issue. Boston: The Weekly Bulletin Publishing Co.

Wiley, J. S., Rasmusen, E., y Ramseyer, J. M. (1990). The leasing monopolist. UCLA Law Review, 37, 693-731. 